\title{
AVALIAÇÃO DO DIAGNÓSTICO DE HIPERTENSÃO ARTERIAL SISTÊMICA NA ATENÇÃO PRIMÁRIA À SAÚDE
}

Thaís Gonçalves de Matos ${ }^{1}$, Waleska Thicyara Cândida dos Santos ${ }^{1}$, Pablo Phillipe Cândido dos Santos ${ }^{2}$, Alex Wander Nenartavis ${ }^{1}$, Jaqueline Costa Castilho Moreira ${ }^{3}$

Universidade do Oeste Paulista - UNOESTE. ${ }^{1}$ Curso de Medicina. ${ }^{2}$ Departamento de Medicina. Presidente Prudente, SP. ${ }^{2}$ Universidade Federal do Amazonas - UFAM. Curso de Medicina. Manaus, AM. ${ }^{3}$ Universidade Estadual - UNESP. Departamento de Educação Física. Presidente Prudente, SP. E-mail: thais.goncalvesm@gmail.com

\section{RESUMO}

A Hipertensão Arterial Sistêmica é considerada um grave problema de saúde por ser fator de risco para Doenças Cardiovasculares, seu diagnóstico e tratamento devem ser realizados pela Atenção Primária à Saúde, responsável pela resolução de aproximadamente $85 \%$ das necessidades de saúde da população. Neste contexto, o objetivo desse estudo foi avaliar a Atenção Primária com relação ao número de diagnósticos de Hipertensão realizados. Trata-se de um estudo descritivo exploratório, de abordagem quantitativa, realizado com 200 usuários atendidos em unidades de atenção básica por meio de entrevistas estruturadas. Os resultados obtidos apontaram uma média de idade de 64,98 anos, sendo que a realização do diagnóstico na Estratégia de Saúde da Família foi de 59\%, e na Unidade Básica de Saúde foi de $43 \%$. Conclui-se desta pesquisa a necessidade de melhoria nos diagnósticos dos serviços de saúde da Atenção Primária para que se evitem maiores agravos aos usuários de saúde hipertensos.

Palavras-chave: hipertensão arterial sistêmica, diagnóstico, atenção primária à saúde, atenção básica, atenção secundária.

\section{DIAGNOSTIC EVALUATION OF SYSTEMIC ARTERIAL HYPERTENSION IN PRIMARY HEALTH CARE}

\begin{abstract}
Systemic Arterial Hypertension is considered a serious health problem for being a risk factor for Cardiovascular Diseases, and its diagnosis and treatment must be carried out by Primary Health Care, responsible for resolving approximately $85 \%$ of the health needs of the population. In this context, the aim of this study was to evaluate the Primary Attention with respect to the number of performed diagnoses of Hypertension. This is an exploratory, descriptive study of quantitative approach carried out with 200 users assisted in primary health care units through structured interviews. The results showed an average age of 64.98 years, and the rate of diagnoses made in the Family Health Strategy was $59 \%$, and $43 \%$ in the Basic Health Unit. To conclude, the results of this research indicate the need for improvement in the diagnostic performance of Primary Care health services to avoid major damages to hypertensive users.
\end{abstract}

Keywords: systemic arterial hypertension, diagnosis, primary health care, basic attention, secondary health care. 


\section{INTRODUÇÃO}

A Hipertensão Arterial Sistêmica (HAS) é uma doença multifatorial caracterizada por níveis elevados e sustentados de pressão arterial ${ }^{1}$, possui longo curso assintomático e evolução clínica lenta ${ }^{2}$, sendo considerada um grande problema de saúde pública por ser fator de risco para doenças cardiovasculares, ${ }^{3}$ e sua prevalência na população idosa se encontrar em torno de $55 \%$ em todas as regiões geográficas do Brasil ${ }^{4}$.

Os fatores de risco que contribuem para o desenvolvimento da HAS primária são: idade maior que 40 anos, cor não branca, excesso de peso e obesidade, ingestão de álcool, sedentarismo e fatores genéticos ${ }^{1}$. Entre as complicações mais frequentes, podemos destacar alterações de órgãos alvo, como o coração, encéfalo, rins e vasos sanguíneos; e alterações metabólicas, sendo este o principal fator para o Acidente Vascular Encefálico (AVE), Infarto Agudo do Miocárdio (IAM) e Doença Renal Crônica ${ }^{5}$.

O diagnóstico da HAS é feito pela medida casual, mas nem sempre é realizado de forma adequada, sendo necessários o preparo do paciente, uma técnica correta e um equipamento devidamente calibrado. É definido por valores de PA sistólica $\geq 140 \mathrm{mmHg}$ e/ou de PA diastólica $\geq$ $90 \mathrm{mmHg}$ em medidas de consultório, sempre validado por medidas repetidas, em condições ideais, em pelo menos três ocasiões. Além disso, recomenda-se a utilização da MAPA (Monitorização Ambulatorial da Pressão Arterial de 24 horas) e da MRPA (Monitorização Residencial da Pressão Arterial) na investigação de pacientes com suspeita de hipertensão para o esclarecimento do diagnóstico ${ }^{1}$.

Após o diagnóstico, se faz necessária avaliação clínica e laboratorial do hipertenso, visando detectar lesões clínicas ou subclínicas com o objetivo de melhor estratificação do risco cardiovascular, além de avaliar indícios de diagnóstico de Hipertensão Arterial Secundária como: hiperaldosteronismo, feocromocitoma e hipertireodismo ${ }^{1}$.

A HAS pode ser tratada e controlada com mudanças no estilo de vida, com medicamentos de baixo custo e de poucos efeitos colaterais, comprovadamente eficazes e de fácil aplicabilidade na Atenção Primária à Saúde (APS) ${ }^{6}$.

No Brasil, a APS é constituída pela Estratégia de Saúde da Família (ESF) e a Unidade Básica de Saúde (UBS), sendo a porta de entrada do usuário no sistema de saúde ${ }^{7}$ que surgiu como uma proposta de enfrentar as iniquidades sociais e ampliar o acesso aos serviços de saúde ${ }^{8}$. A APS representa um complexo conjunto de conhecimentos e procedimentos e exige uma intervenção ampla em diversos aspectos para que se possa ter efeito positivo sobre a qualidade de vida da população ${ }^{9}$.

A Atenção Secundária é formada por serviços ambulatoriais com médicos especializados e atendimentos de urgência e emergência ${ }^{10}$. É nesse nível atenção que são atendidas condições em que há elevação crítica da pressão arterial com quadro clínico grave, progressiva lesão de órgãosalvo e risco iminente de morte ${ }^{6}$.

Neste contexto, o objetivo desse estudo foi avaliar a Atenção Primária com relação ao número de diagnósticos de Hipertensão realizados.

\section{METODOLOGIA}

Trata-se de um estudo descritivo exploratório, de abordagem quantitativa, realizado com usuários cadastrados e atendidos em uma ESF e em uma UBS. Este é um recorte do trabalho intitulado "A relação da presença dos Agentes Comunitários de Saúde na adesão ao tratamento da Hipertensão Arterial Sistêmica", que foi realizado na rede básica de saúde de um município do interior do oeste do estado de São Paulo, de Dezembro 2014 a Maio de 2015. O trabalho foi aprovado pelo Comitê de Ética em Pesquisa da Universidade do Oeste Paulista, sob o protocolo de número 2141 e CAAE número 32317114.2.0000.5515, sendo respeitadas as diretrizes da Resolução 466/2012 do Conselho Nacional de Saúde. 
Para compor o estudo, foram selecionados 100 usuários de cada Unidade, sendo 50 do sexo feminino e 50 do sexo masculino em cada uma delas. Os critérios para participação no estudo foram ter idade igual ou superior a 50 anos, ser usuário de tratamento medicamentoso para hipertensão arterial há pelo menos um ano, participarem ou não dos grupos de apoio das unidades e, finalmente, concordarem e assinarem o Termo de Consentimento Livre e Esclarecido (TCLE). Os dados da pesquisa foram obtidos por meio de visitas domiciliares aos usuários, e de entrevistas nas salas de espera para atendimento médico nas Unidades.

A caracterização da amostra foi realizada por meio da coleta das seguintes variáveis: idade, sexo, cor/raça, estado civil e escolaridade. Além disso, os participantes foram questionados acerca do local onde receberam o diagnóstico de HAS, se este foi realizado na Atenção Primária ou na Atenção Secundária à Saúde. Infere-se que o atendimento na Atenção Secundária está relacionado a complicações decorrentes da HAS. As demais respostas que não se incluíam nos atendimentos citados foram classificadas como "Demais atendimentos", dentre elas, Atendimento com Médico Especialista ou Ambulatorial e Mutirões.

A análise dos dados foi realizada por meio de estatística descritiva, com apresentação dos resultados, em freqüência absoluta e relativa, sendo todos os procedimentos realizados no software BioEstat 5.0.

\section{RESULTADOS}

Do total de hipertensos estudados, encontrou-se uma média de idade de 64,98 anos, com pouca diferença entre as unidades, sendo que a ESF apresentou uma média de 64,73, enquanto na UBS a média de idade foi de 65,22 anos. Com relação à cor, houve predomínio de brancos $(44,5 \%)$ e pardos (33\%), e uma minoria de cor preta (22\%). Quanto à escolaridade, a maioria apresenta ensino fundamental completo e/ou ensino médio incompleto $(72,5 \%)$, em comparação à analfabetos (12,5\%), ensino médio completo (10,5\%) e graduação e/ou pós-graduação (4,5\%). Quanto ao estado civil, $59,5 \%$ dos hipertensos são casados e $40,5 \%$ são solteiros, viúvos, separados ou divorciados. Na TABELA 1 é possível observar a distribuição das frequências absoluta e relativa para ambas as unidades.

Em relação ao local onde receberam o diagnóstico de HAS, 59\% dos usuários da ESF indicaram a APS, 29\% afirmaram receber o diagnóstico na Atenção Secundária, enquanto 12\% relataram outros locais. Dados bem semelhantes aos da UBS, e sem diferença significativa, onde 43\% dos hipertensos relataram ter recebido o diagnóstico na Atenção Primária, 35\% na Atenção Secundária e $22 \%$ em outro local (FIGURA 1). 
Tabela 01. Características biossociais dos hipertensos atendidos pela Estratégia de Saúde da Família - ESF, e pela Unidade Básica de Saúde - UBS, Presidente Prudente, 2014 a 2015.

\begin{tabular}{|c|c|c|c|c|c|c|}
\hline \multirow[b]{2}{*}{ CARACTERÍSTICA } & \multicolumn{2}{|c|}{ ESF } & \multicolumn{2}{|c|}{ UBS } & \multicolumn{2}{|c|}{ TOTAL } \\
\hline & $\begin{array}{l}\text { Freq. } \\
\text { Abs. }\end{array}$ & $\%$ & $\begin{array}{l}\text { Freq. } \\
\text { Abs. }\end{array}$ & $\%$ & $\begin{array}{l}\text { Freq. } \\
\text { Abs. }\end{array}$ & $\%$ \\
\hline \multicolumn{7}{|l|}{ COR/RAÇA } \\
\hline Branca & 36 & 36,0 & 53 & 53,0 & 89 & 44,5 \\
\hline Preta & 29 & 29,0 & 15 & 15,0 & 44 & 22,0 \\
\hline Amarelo & 0 & 0,0 & 1 & 1,0 & 1 & 0,5 \\
\hline Pardo & 35 & 35,0 & 31 & 31,0 & 66 & 33,0 \\
\hline \multicolumn{7}{|l|}{ ESCOLARIDADE } \\
\hline Analfabeto & 18 & 18,0 & 7 & 7,0 & 25 & 12,5 \\
\hline $\begin{array}{c}\text { Ensino Fundamental Completo e } \\
\text { Médio Incompleto }\end{array}$ & 76 & 76,0 & 69 & 69,0 & 145 & 72,5 \\
\hline Ensino Médio Completo & 6 & 6,0 & 15 & 15,0 & 21 & 10,5 \\
\hline Graduação e/ou Pós Graduação & 0 & 0,0 & 9 & 9,0 & 9 & 4,5 \\
\hline \multicolumn{7}{|l|}{ ESTADO CIVIL } \\
\hline Casado & 50 & 50,0 & 69 & 69,0 & 119 & 59,5 \\
\hline Outro & 50 & 50,0 & 31 & 31,0 & 81 & 40,5 \\
\hline
\end{tabular}

*Outro se refere a solteiro, viúvo, separado ou divorciado.

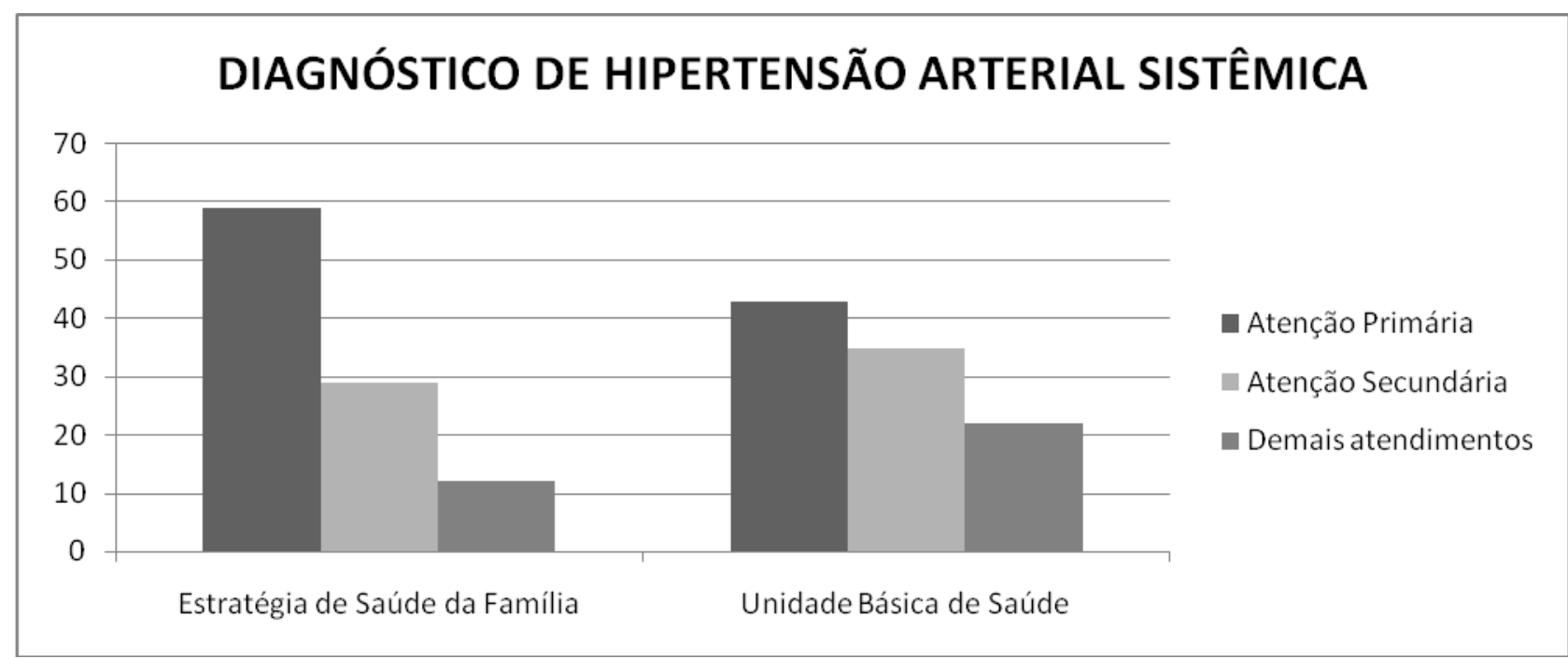

Figura 01. Distribuição de diagnósticos de Hipertensão Arterial Sistêmica, segundo Atenção Primária à Saúde ou Atenção Secundária à Saúde, em ambas unidades de acompanhamento de hipertensos, Presidente Prudente, 2014 a 2015 *Demais atendimentos se refere a Atendimento com Médico Especialista ou Ambulatorial e Mutirões

\section{DISCUSSÃO}

No presente estudo, foi verificado que a maior parte dos diagnósticos de HAS é realizada em serviços de Atenção Primária à Saúde (59\%, no caso da ESF; e 43\%, na UBS). Entretanto há uma porcentagem considerável de usuários do SUS que realizaram o primeiro atendimento para o diagnóstico de HAS em serviços da Atenção Secundária (35\%, no caso da ESF; e 43\%, na UBS), e em Outros Locais (12\%, no caso da ESF; e 22\%, na UBS), conforme mostrado na Figura 1.

Esses dados demonstram que a porcentagem de casos diagnosticados na Atenção Primária ainda não está dentro do desejado. Enquanto porta de entrada do sistema de saúde, a Atenção Primária tem poder de resolução de aproximadamente $85 \%$ das necessidades de saúde da 
população ${ }^{11}$, sendo responsável por serviços preventivos, curativos, reabilitadores e de promoção da saúde. Dessa forma, a APS deve ser capaz de prevenir possíveis problemas de saúde da população e evitar que eles se desenvolvam ou mesmo que gerem maiores complicações.

No entanto, o grande número de diagnósticos realizados na Atenção Secundária aponta para a existência de casos em que o diagnóstico de HAS só é realizado após a ocorrência de complicações da doença, como AVE, IAM, entre outros. Isso se deve a falhas do sistema de busca e diagnóstico de casos de HAS. Como as pessoas acometidas não são diagnosticadas nas fases iniciais da doença, e são encaminhadas quando ela está em uma fase mais avançada, acabam precisando de hospitalização, o que torna os hospitais locais de acesso ao diagnóstico cada vez mais frequentes. Assim, diversos diagnósticos são realizados em dissonância com o princípio da descentralização das ações de saúde pregada pela Política Nacional de Atenção Básica ${ }^{12}$.

Além disso, é possível apontar outras razões que levam os usuários do SUS a escolher serviços médicos da Atenção Secundária em detrimento da Atenção Primária, como as experiências que eles tiveram anteriormente com esses serviços de saúde, como as relacionadas ao horário de funcionamento restrito dos serviços de Atenção Primária; e a aspectos culturais, como a associação entre a estrutura física e tecnológica de hospitais e pronto atendimentos, e a capacidade que eles têm de resolver problemas de saúde.

\section{CONCLUSÃO}

Os resultados da pesquisa indicam que embora a maioria dos diagnósticos de HAS tenham sido realizados nos serviços de saúde da Atenção Primária, esse número ainda não está dentro do ideal determinado pela Política Nacional de Atenção Básica e reflete as falhas na execução do plano de descentralizar as ações de saúde.

Os dados mostram que uma parcela considerável dos diagnósticos de HAS foi realizada em serviços de Atenção Secundária por diversos fatores, como a falha da Atenção Primária em detectar esses casos antes que evoluíssem para complicações, a experiência e a percepção pessoal dos usuários de saúde para com os serviços da Atenção Primária de saúde.

Com base nisso, torna-se premente a necessidade de melhorar o desempenho de diagnósticos dos serviços de saúde da Atenção Primária e evitar maiores agravos aos usuários de saúde. Vale ressaltar que é necessário educar melhor a população quanto ao funcionamento do Sistema Único de Saúde (SUS) para que não tenham receio de serem atendidos pelos serviços de saúde da Atenção Primária.

\section{REFERÊNCIAS}

VI Diretrizes Brasileiras de Hipertensão. Arq. Bras. Cardiol. [Internet]. 2010; 95( 1 Suppl 1 ): I-III. DOI: http://dx.doi.org/10.1590/S0066-782X2010001700001

Manfroi A, Oliveira FA. Dificuldades de adesão ao tratamento na hipertensão arterial sistêmica: considerações a partir de um estudo qualitativo em uma Unidade de Atenção Primária à Saúde. RevBras Med Fam Com. 2006;2(7):165-76. DOI: http://dx.doi.org/10.5712/rbmfc2(7)52

Mendonça LBA, Lima FET, Oliveira SKP. Acidente vascular encefálico como complicação da hipertensão arterial: quais são os fatores intervenientes? Esc Anna Nery. 2012; 16(2): 340-6. DOI: http://dx.doi.org/10.1590/S1414-81452012000200019

Mendes G. Moraes C. Gomes L. Prevalência de hipertensão arterial sistêmica em idosos no Brasil entre 2006 e 2010. Revista Brasileira de Medicina de Família e Comunidade. 2014; 9(32): 273-278. DOI: http://dx.doi.org/10.5712/rbmfc9(32)795 
Lima SA, Gaia MS, Ferreira AM. A Importância do programa HIPERDIA em uma unidade de saúde da família do município de Serra Talhada - PE, para Adesão dos Hipertensos e Diabéticos ao Tratamento medicamentoso e dietético. Saúde Coletiva em Debate. 2012; 2(1):30-29.

Brasil. Ministério da Saúde. Secretaria de Atenção à Saúde. Departamento de Atenção Básica. Estratégias para o cuidado da pessoa com doença crônica: hipertensão arterial sistêmica. Normas e Manuais Técnicos. Cadernos de Atenção Básica; n.37. Brasília: MS; 2013.

Paim JS. Atenção Primária à Saúde: uma receita para todas as estações?. Saúde debate [Internet]. 2012; 36 (94): 343-347. DOI: http://dx.doi.org/10.1590/S0103-11042012000300004

Mitre SM, Andrade EIG, Cotta RMM. Avanços e desafios do acolhimento na operacionalização e qualificação do Sistema Único de Saúde na Atenção Primária: um resgate da produção bibliográfica do Brasil. Ciênc Saúde Colet. 2012;17(8):2071-85. DOI: http://dx.doi.org/10.1590/S1413-81232012000800018

Brasil. Ministério da Saúde. Secretaria de Atenção à Saúde. Departamento de Atenção Básica. Diretrizes do NASF - Núcleo de Apoio à Saúde da Família. Normas e Manuais Técnicos. Cadernos de Atenção Básica; n. 27. Brasília: MS; 2009.

Erdmann AL. Andrade SR. Mello ALSF. Drago LC. A atenção secundária em saúde: melhores práticas na rede de serviços. RevLatinoamEnferm. 2013; 21(esp):131-9. DOI: http://dx.doi.org/10.1590/S0104-11692013000700017

Starfield B. Atenção primária: equilíbrio entre necessidades de saúde, serviços e tecnologia. Brasília: Organização das Nações Unidas para a Educação, a Ciência e a Cultura/Ministério da Saúde; 2002.

Brasil. Ministério da Saúde. Secretaria de Atenção à Saúde. Departamento de Atenção Básica. Política Nacional de Atenção Básica. 4. ed. Brasília: MS; 2007. 